\title{
789 GENERATION OF A BICYCLE NK-TICATM, A NOVEL NK CELL ENGAGING MOLECULE TO ENHANCE TARGETED TUMOR CYTOTOXICITY
}

Fay Dufort*, Christopher Leitheiser, Gemma Mudd, Julia Kristensson, Alexandra Rezvaya, Elizabeth Repash, Eric Haines, Katie Gaynor, Sandra Uhlenbroich, Liudvikas Urbonas, Heather Allen, Helen Harrison, Liuhong Chen, Philip Brandish, Kevin McDonnell, Nicholas Keen. Bicycle Therapeutics, Lexington, MA, United States

Background Natural killer (NK) cells are immune cells that can detect and eliminate tumor cells and bridge innate to adaptive immune responses. Tumor specific activation of NK cells is thus an area of active investigation in immune oncology, but to date has relied on complex biologic modalities (e. g., antibodies, fusion proteins, or cell therapies), each of which has inherent disadvantages in this application. Thus, alternative approaches are warranted. Bicycle ${ }^{\circledR}$ are small (ca. $1.5 \mathrm{kDa}$ ), chemically synthetic, structurally constrained peptides discovered via phage display and optimized using structure-driven design and medicinal chemistry approaches. We have now applied this technology to identify Bicycles that bind specifically to the key activating receptors, NKp46 and CD16a. When chemically coupled to tumor antigen binding Bicycles this results in highly potent, antigen-dependent receptor activation and NK cell activation. We term this new class of fully synthetic molecules Bicycle ${ }^{\circledR}$ natural killer- tumor-targeted immune cell agonists (NK-TICAs ${ }^{\mathrm{TM}}$ ) and we will describe their discovery and evaluation in this presentation.

Methods Using our unique phage display screening platform, we have identified high affinity, selective binders to NKp46 and CD16a. By conjugating the Bicycle ${ }^{\circledR}$ NK cell-engaging binders to a model tumor antigen EphA2-binding Bicycle ${ }^{\circledR}$, we have developed a bifunctional Bicycle NK-TICA ${ }^{\mathrm{rm}}$ molecule. In in vitro functional assays, we evaluated the ability of the Bicycle NK-TICAs ${ }^{\mathrm{TM}}$ to induce NK cell activation as well as cell-mediated cytotoxicity and cytokine production in NKtumor co-culture assays.

Results We have developed a novel modular compound with high affinity and selectivity to NK cell receptors with specific tumor targeting capability. We demonstrate potent, selective binding of our Bicycles to receptor-expressing cells and the capability of the bifunctional molecule to induce NK cell function. With Bicycle's novel NK-TICA ${ }^{\mathrm{TM}}$ compound, we demonstrate engagement of NK cells, specific activation and function of NK cells, and enhanced EphA2-expressing tumor cytotoxicity, in a dose dependent manner.

Conclusions Bicycle NK-TICAs ${ }^{\mathrm{TM}}$ are novel therapeutic agents capable of enhancing the landscape of immune oncology. We hypothesize that utilization of Bicycle NK-TICA ${ }^{\mathrm{TM}}$ as a multifunctional immune cell engager will promote modulation of NK cells, and infiltration and anti-tumor activity of NK cells in solid tumors. The data presented here provide initial proof of concept for application of the Bicycle technology to drive NK cell-mediated tumor immunity.

http://dx.doi.org/10.1136/jitc-2021-SITC2021.789 